\title{
The histopathological effects of intracavernosal mitomycin-C injection in a rat Peyronie's disease model
}

\author{
Engin Kaya ${ }^{1}$; Yusuf Kibar ${ }^{2}$; Sercan Yilmaz ${ }^{1}$; Ayhan Ozcan ${ }^{3}$; Burak Kopru ${ }^{4}$ Turgay Ebiloglu ${ }^{1}$ Hasan Cem Irk1lata ${ }^{1}$ \\ ${ }^{1}$ Gulhane Training and Research Hospital, Department of Urology, Ankara, Turkey; ${ }^{2}$ Korea Hospital, Department of Urology, Ankara, Turkey; ${ }^{3}$ Yeni Yuzyil University, \\ Department of Pathology, Istanbul, Turkey; ${ }^{4}$ Konya Training and Research Hospital, Department of Urology, Konya, Turkey
}

Cite as: Can Urol Assoc J 2017; Epub ahead of print. http://dx.doi.org/10.5489/cuaj.4489

\section{Published online November 1, 2017}

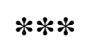

\section{Abstract}

Introduction: We aimed to evaluate whether or not nitomycin-C (MMC) has an anti-fibrotic effect on transforming growth factor beta (TGF- $\beta$ )induced Peyronie's disease (PD) in a rat model.

Methods: Eighteen 12-week-old male Sprague-Dawley rats were divided into three groups: Group 1= TGF- $\beta 1$ (n:7); Group 2= TGF- $\beta 1+$ MMC (n:7); and Group 3= Sham group ( $0.25 \mathrm{ml}$ bovine serum albumin injected) (n:4). All groups were sacrificed on the sixth week of the procedure and their penises were excised. All penis specimens were evaluated semi-quantitatively and quantitatively with histochemical, immunohistochemistry and image analysis.

Results: Both Group 1 and Group 2 had significantly higher fibrosis scores and lower elastic fibers in both outer surface of tunica albuginea (TA) and subsinusoidal area compared with Group 3. When compared with Group 1, the amount of collagen was significantly decreased in Group 2. Intracavernosal MMC injection (Group 2) ended up with lower elastic fibers when compared with Group 1. According to the quantitative analyses, when compared with Groups 1and 3, lower dorsal, ventral, and trabecular thickening values were seen in Group 2. These parameters were only statistically significant when compared with Group 1, suggesting the anti-fibrotic effect of TGF- $\beta 1$-induced fibrosis. Both Groups 1 and 2 showed lower decorin staining levels in SATA and SATW when compared with Group 3. The statistically significant difference was only detected between Group 1 and Group 3.

Conclusions: Our study demonstrates the anti-fibrotic effects of MMC on PD. Further clinical studies are necessary to make inferences regarding its clinical use. 


\section{Introduction}

Peyronie's disease (PD) is characterized by the formation of fibrous plaques in the tunica albuginea (TA). It causes penile curvature, erectile dysfunction and pain during erection[1,2]. The general prevalence of PD is between $3.2 \%$ and $8.9 \%$ [3].

PD contains two phases: acute and chronic. Pain, plaque formation and progression in penile curvature are observed in acute inflammatory phase (6-12 months). Following this period, chronic phase starts and it contains stability of the penile deformity, pain resolution, symptoms of erectile dysfunction (ED) [4].

Trauma is the main etiologic reason of the disease. Repeated microtrauma or macrotraumatic buckling of the rigid phallus cause abnormal wound healing following fibroblast proliferation and collagen deposition [5]. Transforming growth factor beta (TGF- $\beta$ ), a cytokine, has the key role for PD pathogenesis. Expression of TGF- $\beta$ was determined in both human and rat plaque formation [6]. It was showed that successful results were obtained by using anti- TGF- $\beta$ agent in animal models $[7,8]$.

Decorin, an antifibrotic agent, regulates collagen fibrils and mostly expressed by fibroblasts, myocytes and smooth muscle cells [9,10]. It shows the effect by organizing the formation of collagen fibrils and inhibiting TGF- $\beta$ [11]. Decorin staining can be used for evaluating the fibrosis level.

Mitomycin-C (MMC), a chemotherapeutic agent, shows antitumoral and antifibrotic activity. Occleston et al. reported that MMC reduced fibrosis on tenon capsule by effecting TGF- $\beta$ and fibroblast growth factor (FGF). This effect decreased type 1 collagen and fibronectin production [12]. MMC has been used in ophtalmologic, orthopedic and autolaringologic surgery due to its antifibrotic effect [13-15].

In this study, we aimed to evaluate whether or not MMC has an antifibrotic effect on TGF- $\beta$ induced PD in a rat model. To our knowledge, this is the first study evaluating the antifibrotic effect of MMC in PD.

\section{Methods}

This study was performed in accordance with the guidelines of the Experimental Animal Care and approved by the Ethical Committee for Animal Studies at our center. Eighteen 12-week-old male Sprague-Dawley rats (350- 470 g) were divided into 3 groups: Group 1= TGF- $\beta 1$ (n:7), Group 2= TGF- $\beta 1+M M C$ (n:7), and Group 3= Sham group (0.25 ml bovine serum albumin injected) (n:4).

\section{Surgical procedure}

The rats were anesthetized with pentobarbital (35 mg/kg). A small pubic incision was performed to visualize the left proximal section of the TA of the penis in all groups. A total of $0.5 \mu \mathrm{g} / 0.25 \mathrm{ml} \mathrm{TGF- \beta 1} \mathrm{(T} \mathrm{7039,} 0.5 \mu \mathrm{g}$ in a total volume of $0.25 \mathrm{ml}$; Sigma Chemical Co., St. Louis, MO, USA) was injected into TA by a $26-\mathrm{G}$ needle in group 1 and group 2 . A total of $0.25 \mathrm{ml}$ bovine serum albumin, which was used for TGF- $\beta 1$ preparation, was injected in sham group rats. Afterwards, group 2 rats were received intracavernosal MMC (a total of $0.3 \mathrm{mg} / \mathrm{ml}$ ) injection for 
four consecutive days starting from the second day of the TGF- $\beta 1$ injection, and sham group rats were received same doses of intracavernosal saline injection.

All groups were sacrificed on the $6^{\text {th }}$ week of the procedure, and their penises were excised. All penis specimens were evaluated semiquantitatively, and quantitatively using routine and histochemical staining methods, immunohistochemistry and image analysis.

\section{Histopathologic examination}

Following 10\% buffered formalin fixation, all penile specimens was divided into right and left side in the longitudinal axis. After automated tissue processing and afterward cutting procedures, all sections were stained with hematoxylin and eosin (H\&E), Masson's trichrome (to evaluate amount of collagen bundles in TA and trabecular wall, which are stained with blue color, and proportion of smooth muscle cells in same areas, which are stained with red color), and Verhoeff-Van Gieson (to evaluate amount of elastic fibers in subsinusoidal areas of TA and trabecular wall, and subcutaneous connective tissue) methods. For all stained tissue sections, morphological parameters consisted of TA thickening, trabecular wall thickening and sinusoidal narrowing, attenuation or thinning in the smooth muscle layer of TA, increasing of trabecular smooth muscle cells, reduction of elastic fibers in outer of tunica albuginea and sinusoidal wall were semi-quantitatively evaluated by a single pathologist. Thickening of ventral and dorsal TA and sinusoidal narrowing in tissue sections stained with Masson's trichrome were measured under x40 objective using an image analysis program, which is Image J program (Wayne Rasband (wayne@codon.nih.gov)).

\section{a. Semi-quantitative evaluation}

To determine amount of the fibrous tissue and elastic fibers, semi-quantitative examination was performed by a single pathologist using following scoring system: $0=$ No thickening or attenuation or changes; $1(+)=1,25$ times thickening or increasing/thinning or decreasing; $2(+)=$ 1,5 times thickening or increasing/thinning or decreasing; $3(+)=2$ times thickening or increasing/thinning or decreasing (Table 1).

\section{b. Image analysis (quantitative) evaluation}

To determine amount of the fibrous tissue, the all images stained with Masson's trichrome (totally 72 images) that were processed by pathologist were loaded into Image J 10.2 program (http://rsb.info.nih.gov) and all measurements were performed under x40 objective. All measurements were automatically recorded as pixels, and afterward all values were converted to millimeter using a formula.

\section{Statistical analysis}

Statistical analyses were performed using Statistical Package for Social Sciences 20.0 software (SPSS 20.0 for Windows, Chicago). Descriptive statistics were noted with mean, standard deviation, minimum, and maximum. Kurtosis, Skewness, Kolmogorov Simirnov and Shapiro-Wilk 
Tests were used to assess the variables' normalization. Mann-Whitney-U test and Student $t$ test did comparisons of two groups. Comparisons of multiple groups were made by using Mann-Whitney-U testi and Student $t$ test. A probability of $\mathrm{p}<0.05$ was accepted as significant difference.

\section{Results}

\section{Histopathological results}

\section{a. Semiquantitative evaluation}

When compared with group 3 (sham group), previously described semiquantitative scores evaluating fibrosis were higher in both group 1 and group 2, and this finding was statistically significant (Table 1) (Fig. 1a-c). However, these changes regarding collagen amount were significantly decreased in group 2 compared with group 1, suggesting the protective effect of MMC in group 2 (Fig. 2a). Similarly decrease of elastic fibers in both outer surface of TA and subsinusoidal area was significantly higher in both group 1 and group 2 (Table 1) (Fig. 1d-f) Fig.2b).

Intracavernosal MMC injection (group 2) resulted in a decrease of elastic fibers in both TA and sinusoidal wall when compared with group 1, again suggesting the protective effect of MMC against TGF- $\beta 1$ induced fibrosis.

\section{b. Image analysis (quantitative) evaluation}

Group 2 revealed lower dorsal, ventral and trabecular thickening values when compared with group 1 and group 3 (Table 2 and Fig. 2c). This difference was statistically significant when compared with group 1 ( $\mathrm{p}=0.001$ ), suggesting the antifibrotic effect of TGF- $\beta 1$ induced fibrosis. Group 2 and sham group values were similar and the difference was not statistically significant $(\mathrm{p}>0.05)$.

\section{Immunohistochemical analysis}

The amount of decorin staining in subsinusoidal areas of tunica albuginea (SATA) and subsinusoidal areas of trabecular wall (SATW) were summarized in table 1 . While the decorin levels were lower in group 1 and group 2 when compared with group 3, statistically significant difference was observed between group 1 and group 3 (Fig. 1g-i, and Fig. 2d). No staining for decorin in subcutaneous areas (SCA) was detected in both group 1 and group 2 contrary to group 3 (Table 1). In all groups, no TGF- $\beta 1$ staining in SATA, SATW and SCA was observed because of its speed circulation and elimination in cavernous system of penis. All semiquantitative and quantitative analyses and immunohistochemical analysis were summarized in Table 1 and Table 2.

\section{Discussion}

PD is a progressive fibrotic process related with TGF- $\beta 1$, which can cause sexual dysfunction. Several animal models have been reported for PD in the literature. Injection of cytomodulin, fibrin and TGF- $\beta 1$ show fibrotic effect in an animal model as PD. They induce production of TGF- 
$\beta 1[5-7,16]$. In 6 weeks period after injection, chronic cellular infiltration accompany with focal diffuse elastosis, thickening and disorganized collagen fibers and elastin fragmentation [7]. Fibrous plaque, which appears with profibrotic agent injection, is histologically similar to humans' plaque formation [16].

There are some agents used for the treatment of PD in the literature. Anthocyanin extract, anti-inflammatory and antifibrotic agent, was evaluated in terms of treatment of PD in a rat model by Sohn et al [17]. When anthocyanin group compared with only PD group, TGF- $\beta 1$ expressions were decreased statistically significant. The amount of the TGF- $\beta 1$ positive cells in PD group was higher than control and anthocyanin group [17]. In another study, decorin, antifibrotic agent, was administered into the corpus cavernousum in a rat model. Thickening of TA was identified in PD group and PD+decorin injection group as 87.5\%, 37.5\%, respectively. It was reported that decorin protected erectile response via inhibiting TGF- $\beta 1[7]$.

Clostridium Collegenase (CC) injection, which is only medical treatment agent, is approved by United States Food and Drug Administration. In Gelbard et al. study, CC treatment showed significant benefit for penile curvature and outcomes of symptom bother scores [18]. Repeated injections of intralesional verapamil reported to lead to regression in 78\% of patients with minimal side effects in the early stages of the PD [19]. Trost et al. evaluated 127 patients in terms of the effects of interferon- $\alpha 2 \mathrm{~B}$. Intralesional therapy with interferon- $\alpha 2 \mathrm{~b}$ provided significantly improved curvature without impacting penile vascular parameters [20].

MMC is a potent inhibitor of fibroblast besides being widely used chemotherapeutic agent. This effect occurs via inducing apoptosis [21]. MMC has been used various treatment modalities in urology practice except bladder cancer. It was reported that MMC reduced the recurrence rate of urethral stricture after internal uretrotomy [22-23]. In another study, MMC decreased hemosiderin-laden macrophages, mononuclear cell infiltration and fibrosis after internal ureterotomy compared with control groups in a rat model [24].

In our study, we evaluated the antifibrotic effects of MMC on PD. Both group 1 and group 2 had significantly higher fibrosis scores compared with sham group (Group 3). When compared group 1, the amount of collagen were significantly decreased in group 2. At the same time, decrease of elastic fibers in both outer surface of TA and subsinusoidal area was significantly higher in both group 1 and group 2. Intracavernosal MMC injection (group 2) ended up lower elastic fibers in both TA and sinusoidal wall when compared with group 1. This findings were supporting the protective effect of MMC against TGF- $\beta 1$ induced fibrosis.

According to the quantitative analyses, when compared with group 1 and group 3, lower dorsal, ventral and trabecular thickening values were seen in group 2 . These parameters were only statistically significant when compared with group 1, suggesting the antifibrotic effect of TGF$\beta 1$ induced fibrosis. The difference was not statistically significant when compared control group.

Both group 1 and group 2 showed lower decorin staining levels in SATA and SATW when compared with group 3. The statistically significant difference was only detected between group 1 and group 3. No staining for decorin in SCA was observed in both group 1 and group 2 contrary 
to group 3. Although there was no statistically significant differences, lower decorin staining was detected in MMC against TGF- $\beta 1$ induced fibrosis.

The fact that amount of collagen bundles decreased and elastic fibers increased in MMC group (group 2) compared TGF- $\beta 1$ group (group 1) was demonstrated by histopathological (semi-quantitative) and image analysis program (quantitative). These results were statistically significant as mentioned above.

\section{Conclusion}

MMC showed antifibrotic effect on rat PD model. Although new animal and further clinical studies are warranted to make some inferences regarding its clinical use, our study has the proper pride to be the first step of this time course. 


\section{References}

1. Alenda O, Beley S, Ferhi K, et al. Pathophysiology and management of Peyronie’s disease in adult patients: an update. Prog Urol. 2010 Feb;20 (2):91-100.

2. Salem EA, Elkady EH, Sakr A, et al. Lingual mucosal graft in treatment of Peyronie disease. Urology. 2014;84(6):1374-7.

3. Schwarzer U, Sommer F, Klotz T, et al. The prevalence of Peyronie’s disease: results of a large survey. BJU Int. 2001 Nov;88:727730.

4. Greenfield JM, Levine LS. Peyronie’s disease: etiology, epidemiology issepiand medical treatment. Urol Clin North Am. 2005;32:469478.

5. Devine CJ Jr, Somers KD, Jordan SG, et al. Proposal: trauma as the išlicicause of the Peyronie’s lesion. J Urol. 1997 Jan;157(1):285290.

6. El-Sakka AI, Hassoba HM, Pillarisetty RJ, et al. Peyronie’s disease is associated with an increase in transform- ing growth factor-beta protein expression. J Urol 1997; 158:1391-4.

7. Akman T, Tefekli A, Armagan A, et al. Decorin as a new treatment alternative in Peyronie's disease: preliminary results in the rat model. Andrologia. 2013;45(2):101-6.

8. Ryu JK, Piao S, Shin HY, Choi MJ, Zhang LW, Jin HR, et al. IN-1130, a novel transforming growth factor-beta type I receptor kinase (activin receptor-like kinase 5) inhibitor, promotes regression of fibrotic plaque and corrects penile curvature in a rat model of Peyronie's disease. J Sex Med 2009;6:1284-96.

9. Hocking AM, Shinomura T, McQuillan DJ. Leucine-rich repeat glycoproteins of the extracellular matrix.Matrix Biol. 1998 Apr;17(1):1-19. Review.

10. Thieszen SL, Rosenquist TH. Expression of collagens and decorin during aortic arch artery development: implications for matrix pattern formation. Matrix Biol. 1995 Jul;14(7):573-82.

11. Kuc IM, Scott PG. Increased diameters of collagen fibrils precipitated in vitro in the presence of decorin from various connective tissues. Connect Tissue Res. 1997;36(4):287-96.

12. Occleston NL, Daniels JT, Tarnuzzer RW, et al. Single exposures to antiproliferatives: long-term effects on ocular fibroblast woundhealing behavior. Invest Ophthalmol Vis Sci. 1997;38(10):1998-2007.

13. Lee JY, Stenzel W, Impekoven P, et al. The effect of mitomycin C in reducing epidural fibrosis after lumbar laminectomy in rats. J Neurosurg Spine. 2006;5: 53-60.

14. Cincik H, Güngö, A, Cekin E, et al. Effects of topical application of mitomycin-C and 5-fluorouracil onmyrngotomy in rats. Otol Neurotol. 2005;26: 351-354. 
15. Kim JH, Kim MJ, Kim DY, et al. Recurrent corneal hypertrophic scar after laser-assisted subepithelial keratectomy with mitomycin C treatment. Cornea. 2011;30: 1030-1034.

16. Davila HH, Ferrini MG, Rajfer J, et al. Fibrin as an inducer of fibrosis in the tunica albuginea of the rat: a new animal model of Peyronie’s disease. BJU Int. 2003;91:830-838.

17. Sohn DW, Bae WJ, Kim HS, et al. The anti-inflammatory and antifibrosis effects of anthocyanin extracted from black soybean on a Peyronie disease rat model. Urology. 2014;84(5):1112-6.

18. Gelbard M, Lipshultz LI, Tursi J, et al. Phase 2b study of the clinical efficacy and safety of collagenase Clostridium histolyticum in patients with Peyronie disease. J Urol. 2012;187(6):2268-74.

19. Wolff B, Peyronnet B, Cattarino S, et al. Intralesional Injections for Early Peyronie Disease: Standardized Assessment and Analysis of Predictive Factors for Treatment Response. Urology. 2015 Jul;86(1):57-61.

20. Trost LW, Ates E, Powers M, et al. Outcomes of intralesional interferon- $\alpha 2 \mathrm{~B}$ for the treatment of Peyronie disease. J Urol. 2013;190(6):2194-9.

21. Kim TI, Choi SI, Lee HK, et al. Mitomycin C induces apoptosis in cultured corneal fibroblasts derived from type II granular corneal dystrophy corneas. Mol Vis. 2008;14:1222-8.

22. Ali L, Shahzad M, Orakzai N, et al. Efficacy of mitomycin C in reducing recurrence of anterior urethral stricture after internal optical urethrotomy. Korean J Urol. 2015;56(9):650-5.

23. Mazdak H, Meshki I, Ghassami F. Effect of mitomycin C on anterior urethral stricture recurrence after internal urethrotomy. Eur Urol. 2007;51(4):1089-92;

24. Ayyildiz A, Nuhoglu B, Gülerkaya B, et al. Effect of intraurethral Mitomycin-C on healing and fibrosis in rats with experimentally induced urethral stricture. Int J Urol. 2004;11(12):1122-6. 


\section{Figures and Tables}

Fig. 1. $(\boldsymbol{a}, \boldsymbol{b}, \boldsymbol{c})$ The thickening of tunica albuginea and trabecular wall, and sinusoidal narrowing in group 1 is slightly more than groups 2 and 3 (Masson's trichrome stain, $\mathrm{x} 40) .(\boldsymbol{d}, \boldsymbol{e}, \boldsymbol{f})$ The amount of elastic fibers in outer surface of TA and susinusoidal area in group 1 is lower than groups 2 and 3, whereas the amount of elastic fibers in group 2 is almost similar to group 3 (Verhoeff-Van Gieson's elastic stain, x200). (g, $\boldsymbol{h}, \mathbf{i})$ Decorin staining in subsinusoidal areas of tunica albuginea (SATA) and trabecular wall (SATW) in groups 1 and 2 is lower than group 3. No decorin staining is observed in subcutaneous connective tissue of groups 1 and 2 unlike group 3 (Immunohistochemistry, x100).

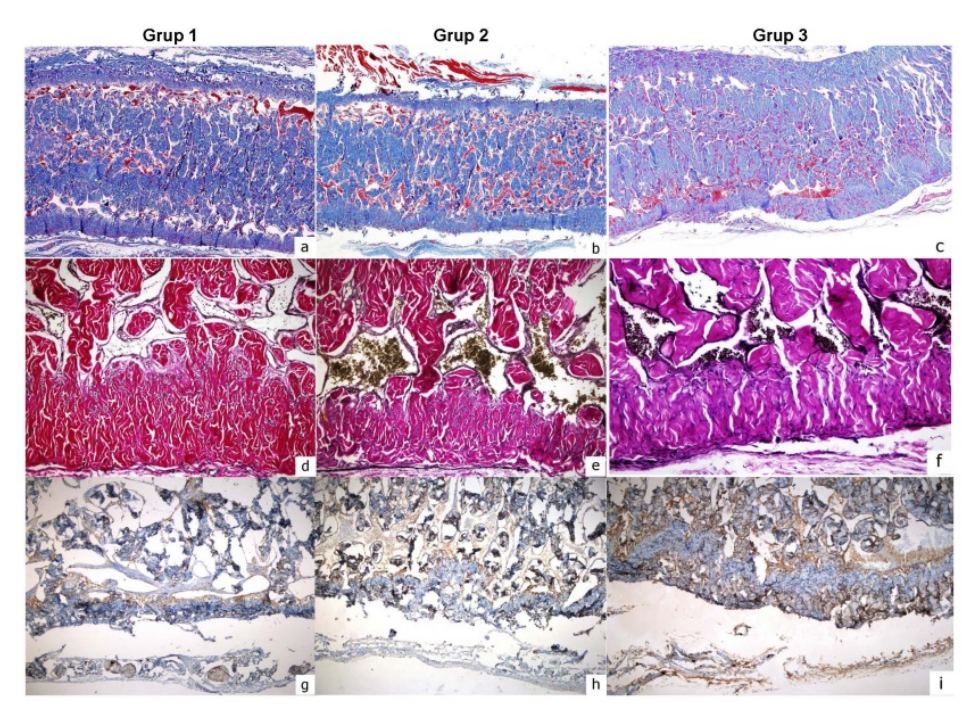


Fig. 2. (a) Histopathological changes in tunica albuginea and trabecular wall among groups. (b) The distribution and amount of elastic fibers among groups. (c) The results of quantitative analysis of the thickness of tunica albuginea and trabecular wall among groups. (d) The proportion of decorin staining in subsinusoidal areas of tunica albuginea (SATA), subsinusoidal areas of trabecular wall (SATW), subcutaneous areas (SCA) among groups.

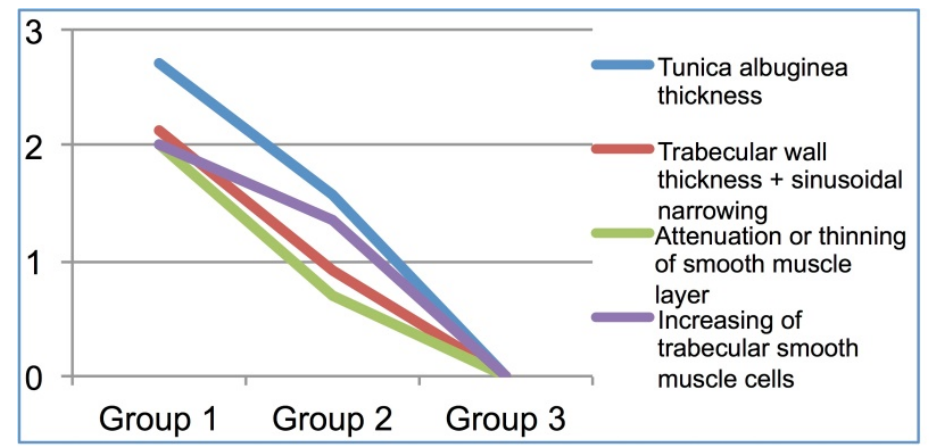

Figure 2a Histopathological changes in tunica albuginea and trabecular wall among groups

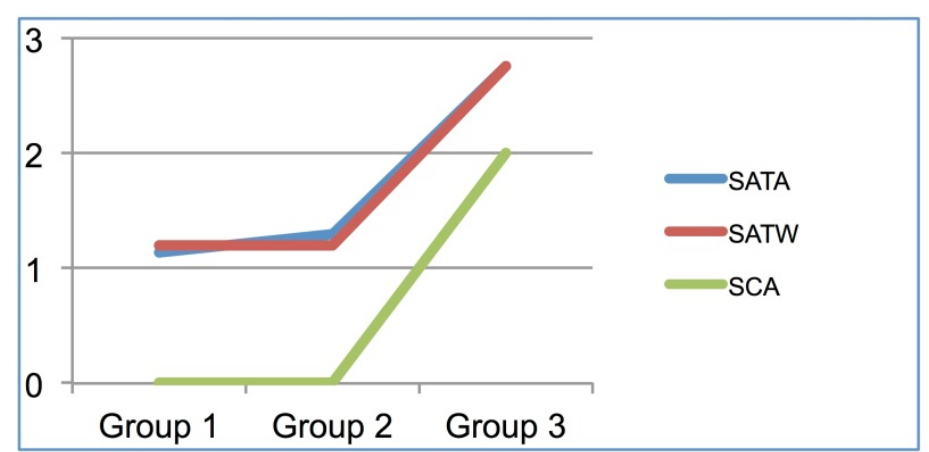

Figure 2c The proportion of decorin staining in subsinusoidal areas of tunica albuginea (SATA), subsinusoidal areas of trabecular wall (SATW), subcutaneous areas (SCA) among groups

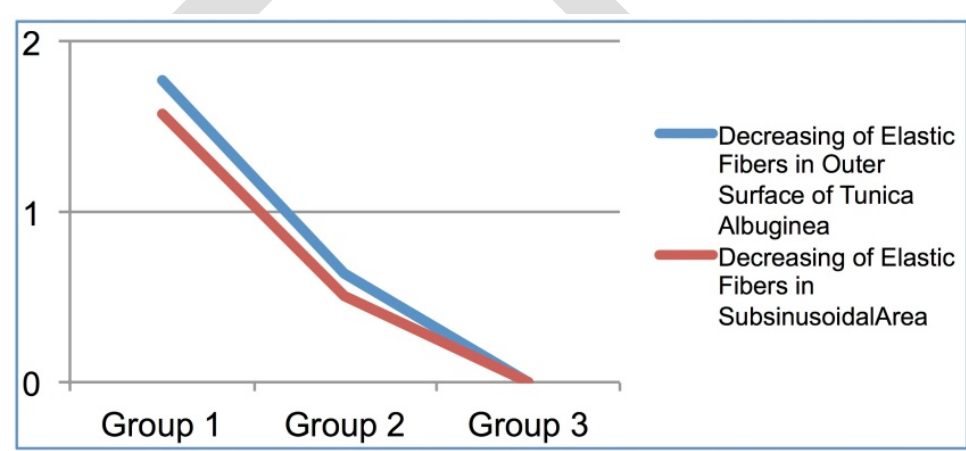

Figure $2 b$ The distribution and amount of elastic fibers among groups

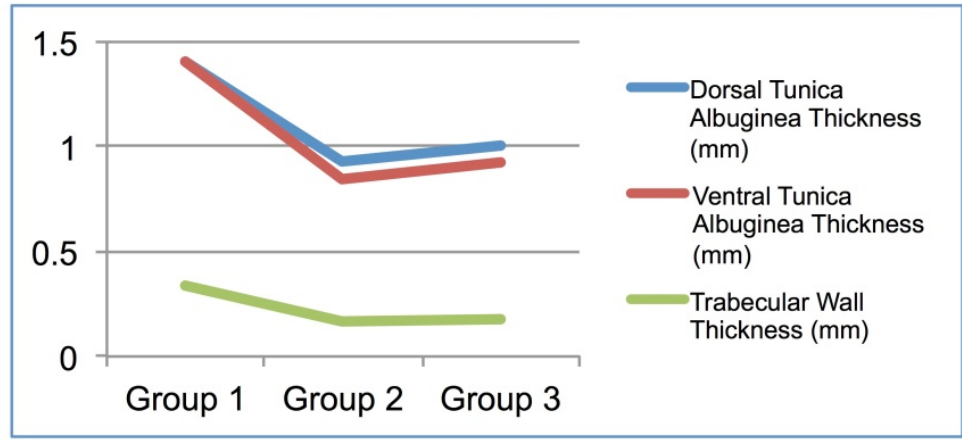

Figure $2 \mathrm{~d}$ The results of quantitative image analysis of the thickness of tunica albuginea and trabecular wall among groups 
Table 1. The results of semi-quantitative and statistical analyses of morphological changes among groups

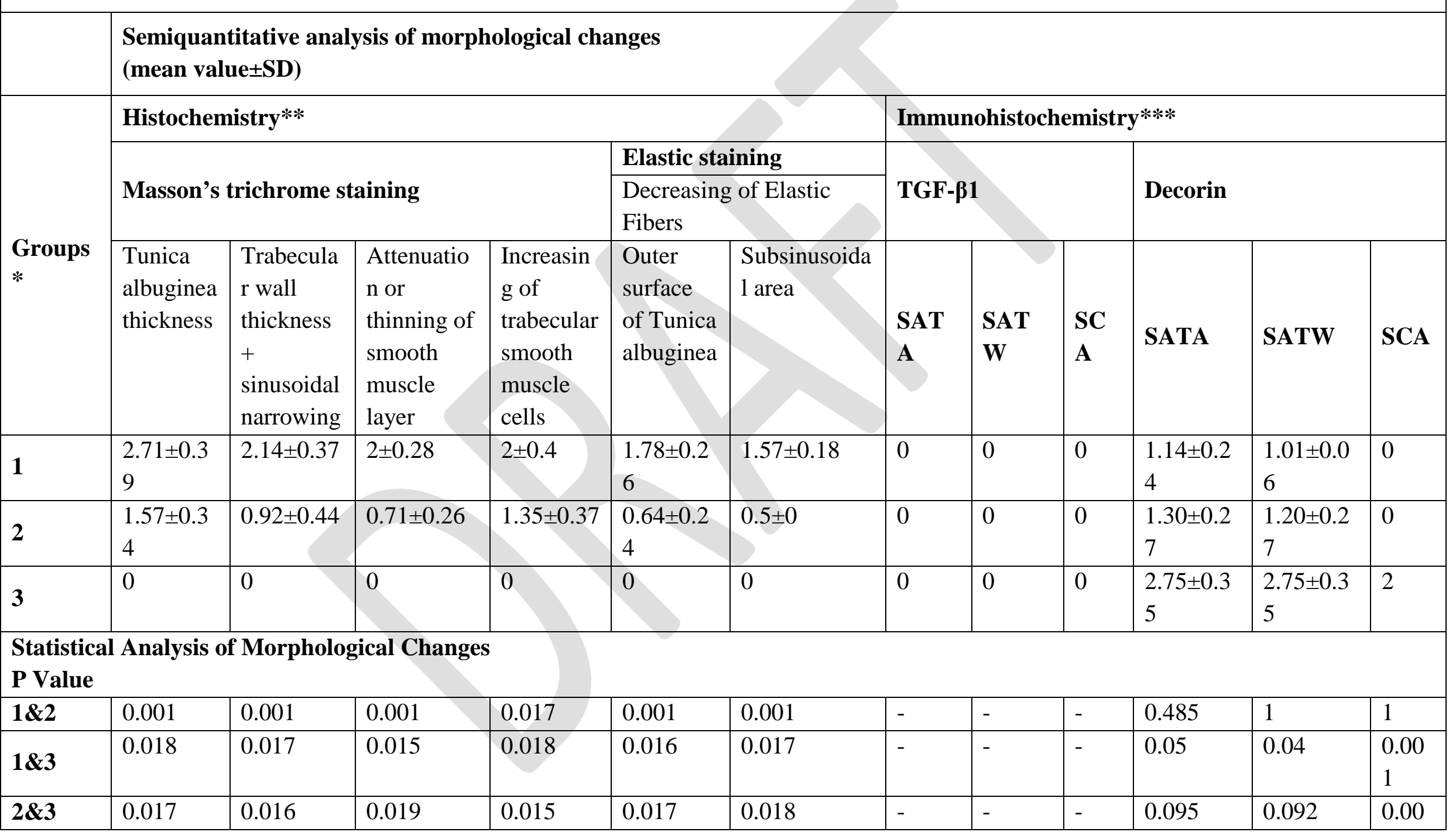




\begin{tabular}{|l|l|l|l|l|l|l|l|l|l|l|}
\hline & & & & & & & & & & \\
\hline
\end{tabular}

* Group 1= TGF- $\beta 1$ (n:7), Group 2= TGF- $\beta 1+M M C$ (n:7), and Group 3= Sham group (n:4). **Scoring for histochemistry: 0= No thickening or attenuation or changes/thinning or decreasing; $1(+)=1,25$ times thickening or increasing/thinning or decreasing; $2(+)=1,5$ times thickening or increasing/thinning or decreasing; $3(+)=2$ times thickening or increasing/thinning or decreasing. *** Scoring for immunohistochemistry: $0=$ no staining, $1(+)$ = weak, $2(+)=$ moderate, $3(+)=$ strong. SATA: Subsinusoidal areas of tunica albuginea; SATW: Subsinusoidal areas of trabecular wall; SCA: Subcutaneous areas

\begin{tabular}{|l|l|l|l|}
\hline \multicolumn{2}{|l|}{ Table 2. The results of quantitative and statistical analyses of morphological changes among groups } \\
\hline \multirow{2}{*}{$\begin{array}{l}\text { Groups } \\
\text { Quantitative results of morphological changes } \\
\text { (mean value } \pm \text { SD) }\end{array}$} & \begin{tabular}{l} 
Ventral TA thickness (mm) \\
\cline { 2 - 4 }
\end{tabular} & & Trabecular wall thickness (mm) \\
\hline $\mathbf{1}$ & $1.4 \pm 0.17$ & $1.4 \pm 0.61$ & $0.3371 \pm 0.02$ \\
\hline $\mathbf{2}$ & $0.93 \pm 0.16$ & $0.84 \pm 0.09$ & $0.1671 \pm 0.01$ \\
\hline $\mathbf{3}$ & $1 \pm 0.18$ & $0.93 \pm 0.18$ & $0.1766 \pm 0.01$ \\
\hline $\begin{array}{l}\text { Statistically analysis of quantitative results of morphological changes } \\
\mathbf{P} \text { value }\end{array}$ & & & \\
\hline $\mathbf{1} \mathbf{2}$ & 0.001 & 0.001 & 0.001 \\
\hline $\mathbf{1} \mathbf{3}$ & 0.017 & 0.117 & 0.017 \\
\hline $\mathbf{2 \& 3}$ & 0.517 & 0.667 & 0.383 \\
\hline
\end{tabular}

